\title{
Chaperon solvent plug to enhance protein refolding in size exclusion chromatography
}

\author{
Hwai-Shen Liu*, Che-Kuei Chang \\ Department of Chemical Engineering, National Taiwan University, Taipei, Taiwan, ROC
}

Received 15 January 2003; accepted 8 April 2003

\begin{abstract}
Size exclusion chromatography (SEC) is an effective way to refold denatured proteins. However, due to the contact between the refolding buffer (mobile phase) and the injected denatured protein (sample), immediate aggregation from injector and column inlet was found to be a barrier of effective refolding. A chaperon solvent plug strategy was, therefore, developed to overcome this problem. That is, the denatured protein was escorted from the injector into the column by a solvent plug that could inhibit formation of aggregates. The refolding of denatured lysozyme in SEC by this method was experimentally shown to reduce the aggregation and, thus, to enhance both mass and activity recoveries simultaneously.
\end{abstract}

(C) 2003 Elsevier Inc. All rights reserved.

Keywords: Chaperon solvent plug; Size exclusion chromatography; Protein refolding; Lysozyme

\section{Introduction}

Expression of genetically engineered proteins in bacteria often results in inactive aggregates inside of the cells, i.e. inclusion bodies. Although recovery of these aggregates requires additional steps, formation of inclusion bodies can be sometimes advantageous [1]. For example, these proteins may be protected against proteolytic degradation when embedded in aggregates. Also, downstream processing may be facilitated since the product could be easily separated from other cellular components by centrifugation or microfiltration. Usually the recovery of the active protein from inclusion bodies involves following steps: isolation and washing, solubilization, and refolding of the denatured protein [2]. While the efficiency of the first two steps can be relatively high, the renaturation yield is very often limited by the accumulation of off-pathway species, such as aggregates [3].

Refolding of denatured proteins is frequently achieved by removing excess denaturants to provide a favorable environment for the inactive proteins to fold into an active form. Methods by dilution [4] and dialysis [5] frequently reported in literature are typical examples. However, partially folded proteins or intermediate products are subject to aggregation

\footnotetext{
* Corresponding author.

E-mail address: hsliu@ccms.ntu.edu.tw (H.-S. Liu).
}

during the refolding process because of their exposed hydrophobic patches. And this could significantly reduce the refolding performance. Thus, how to reduce the aggregation during the refolding process would be an important task. Several methods have been suggested to improve renaturation yield, such as binding the unfolded protein on a strong anion-exchange resin [6], and passing through a column with immobilized molecular chaperones [7].

Refolding by size exclusion chromatography (SEC) has been demonstrated to be an effective way for protein renaturation and attracted some attentions recently [8-12]. The mechanisms of protein refolding inside the SEC column were proposed previously [8]. Mainly, there are two important aspects inside of the SEC column to assist folding. First, the unfolded protein can be easily separated from denaturants by the SEC packing due to their large difference in size. This provides the basis for protein renaturation in refolding buffer (mobile phase) since the denaturant is removed. Secondly, partially folded proteins, isolated within the pores of packing media, could proceed refolding autonomously and segregately. Although this technique may provide not only good refolding but also purification in one step potentially, the process parameters have not been completely elucidated yet. Also, it is suspected that aggregation could occur immediately after injected denatured protein (sample) contacts the refolding buffer (mobile phase). That is, during sample traveling from injector to the column 
inlet, aggregates could be possibly already formed irreversibly and could not properly refold later after entering the SEC column. This can greatly reduce the refolding efficiency. Some hints can also be noted in literature. For example, Fahey et al. [13] noticed that aggregation was crucial in a SEC operation. An urea gradient gel filtration system was suggested to enhance the refolding efficiency [14]. Therefore, a "chaperon solvent plug" strategy was demonstrated in this report to inhibit the aggregates formation before the sample entering the column. This chaperon solvent plug was designed to escort the denatured protein (injected sample) from the injector into column under "aggregation inhibition condition", thus to hopefully enhance protein folding yield.

It has been demonstrated that moderate concentration of urea $(1-2 \mathrm{M})$ in the refolding buffer could enhance protein renaturation yield $[15,16]$. But if the concentration of urea is higher than $3 \mathrm{M}$, it could not improve the activity recovery since it stabilized the proteins in a denatured state [17]. Actually, urea of high concentrations (6-8 M) was used to solubilize aggregates $[1,18]$. This is simply because urea of high concentration could form hydrogen bonds with the protein and these hydrogen bonds would disrupt intermolecular interactions among hydrophobic regions of partially folded proteins [17]. For example, Fahey et al. [13] noticed that the aggregates formation was completely suppressed with urea of $3 \mathrm{M}$ or higher in a batch refolding process by dilution. Nölting [18] also thought that the unfolded protein and aggregates could be solubilized at very high concentration of urea. Therefore, solvent plug of $8 \mathrm{M}$ urea was chosen as the "chaperon" to escort the denatured protein into the column in this investigation.

\section{Experimental}

\subsection{Materials}

Hen egg white lysozyme (HEWL; EC 3.2.1.17) was purchased from Merck. Dithiothreitol (DTT), Micrococus lysodeikitus dried cells, oxidized glutathione (GSSG) and reduced glutathione (GSH) were purchased from Sigma. Urea was purchased from Hayashi Pure Chemical Ind. Co., Ltd. All other chemicals were of analytical grade. Native lysozyme (HEWL) was denatured by incubating the protein $(5 \mathrm{~g} / \mathrm{l})$ in $0.1 \mathrm{M}$ Tris- $\mathrm{HCl}, \mathrm{pH} 8.6$ containing $8 \mathrm{M}$ urea and $0.01 \mathrm{M}$ DTT for $24 \mathrm{~h}$ at room temperature. The activity of denatured lysozyme was found to be zero after incubation.
(A)
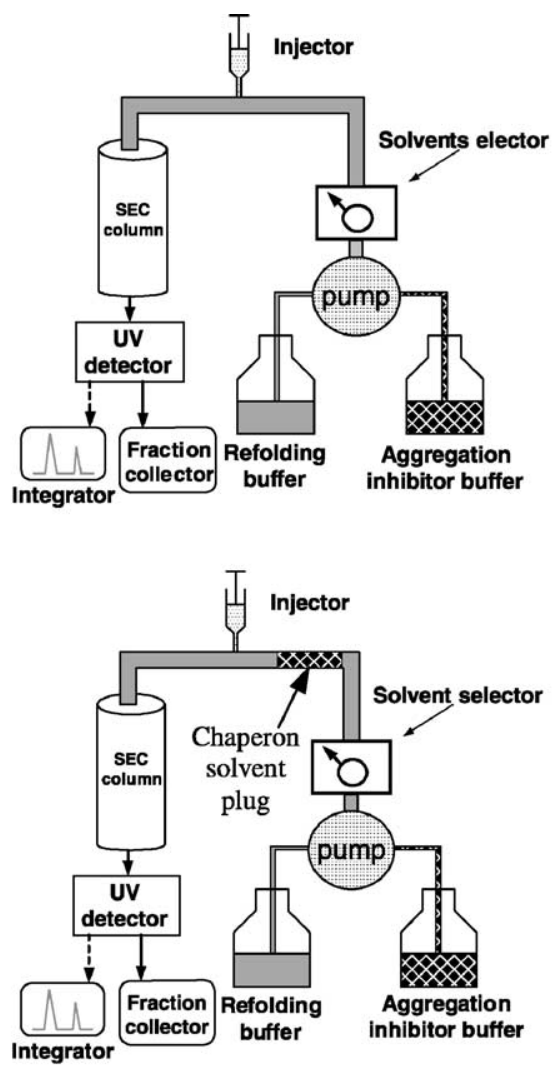

(B)
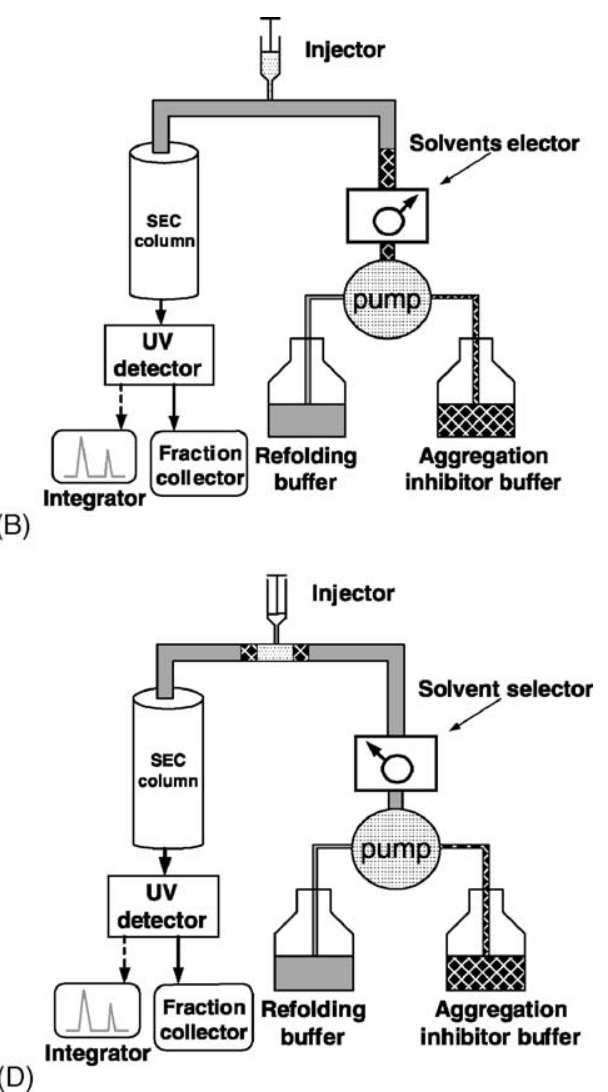

Fig. 1. Illustration of SEC refolding process with the chaperon solvent plug strategy. (A) The column was initially equilibrated with refolding buffer. (B) Aggregation inhibitor solvent was introduced. (C) The chaperon solvent plug was traveled toward injector. (D) As the chaperon solvent plug arrived at injector, denatured protein was injected on the chaperon solvent plug. 


\subsection{Refolding of lysozyme with the chaperon} solvent plug strategy

\subsubsection{High performance liquid chromatography}

Fig. 1 shows the experimental setup and the operation strategy for protein refolding by SEC. The high performance liquid chromatography (Perkin-Elmer) was equipped with a Superdex 75 HR 10/30 column (Pharmacia Biotech), a dual $\lambda$ absorbance detectors (Perkin-Elmer 2487) and a binary pump (Perkin-Elmer 1525). The mobile phase was filtrated by nitrocellulose membrane $(0.2 \mu \mathrm{m})$ and degassed beforehand. The effluent from the column was measured at $280 \mathrm{~nm}$ for protein. Samples were also collected for the enzyme activities analysis. Mobile phase was a refolding buffer $(0.1 \mathrm{M}$ Tris-HCl, pH 8.2, 3 mM GSH, 0.3 mM GSSG, 1.5 M sodium chloride, $1 \mathrm{mM}$ EDTA and $2 \mathrm{M}$ urea) and an aggregation inhibitor solvent containing the same constituents as the refolding buffer of which urea concentration was increased to $8 \mathrm{M}$.

\subsubsection{Operation strategy}

The Superdex 75 HR 10/30 column (Pharmacia Biotech) was initially equilibrated with refolding buffer (Fig. 1A, please note the solvent selector). Then the solvent selector was switched from the mobile phase (refolding buffer) to the aggregation inhibitor solvent (Fig. 1B) for $20 \mathrm{~s}$ and switched back. Therefore, at $1 \mathrm{ml} / \mathrm{min}$ rate, about $333 \mu \mathrm{l}$ solvent plug was introduced into the mobile phase (Fig. 1C). As the chaperon solvent plug arrived at injector, $20 \mu \mathrm{l}$ sample of denatured lysozyme was injected into this solvent plug and moved together into the column inlet (Fig. 1D). The main idea in the strategy was to escort the injected sample into the column; thus avoiding the contact of the denatured protein with refolding buffer and also stabilizing the sample in the segregated form. Obviously, when to inject the denatured protein (sample) into the chaperon solvent plug would be crucial in this strategy. Actually this can be determined by measuring the difference in retention times between known samples introduced from the injector and reservoir of aggregation inhibitor buffer, respectively. This was predetermined as $7.5 \mathrm{~min}$ at $1.0 \mathrm{ml} / \mathrm{min}$ elution rate. In other words, the sample should be injected at $7.5 \mathrm{~min}$ after the chaperon solvent plug was introduced.

\subsection{Analytical methods}

Lysozyme concentration was measured spectrophotometrically at $280 \mathrm{~nm}$. The activity of refolded lysozyme was determined by measuring the absorbance decrease $(450 \mathrm{~nm})$ of $2.5 \mathrm{ml}$ M. lysodeikitus suspension $(0.2 \mathrm{~g} / \mathrm{l})$ in $0.06 \mathrm{M}$ potassium phosphate at $\mathrm{pH} 6.2$ and $25^{\circ} \mathrm{C}$ with $40 \mu$ of protein sample. One unit of activity corresponds to an absorbance decrease of 0.0026 per minute.

Fractions were collected and analyzed for the activities of eluted lysozyme. Mass recovery is defined as the ratio

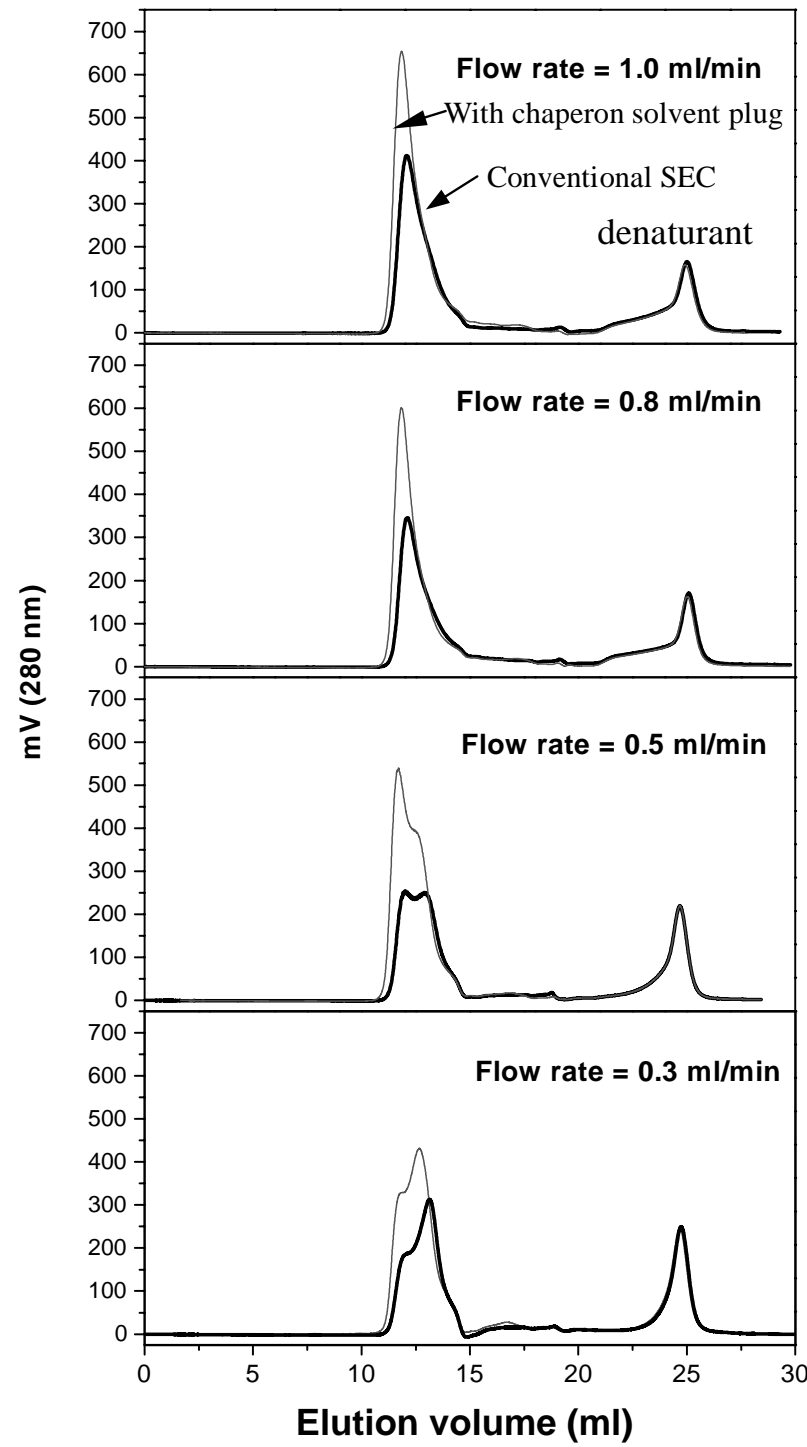

Fig. 2. Elution profiles of conventional SEC refolding process (_) and of the chaperon solvent plug method (_). (Denatured lysozyme loading: $20 \mu \mathrm{l}$ of $5 \mathrm{~g} / \mathrm{l}$.)

of amount of eluted lysozyme to that of injected denatured protein, which was calculated from chromatograms.

\section{Results}

\subsection{Effect of mobile phase flow rate on lysozyme refolding in conventional SEC}

Lysozyme refolding in SEC was experimentally investigated for mobile phases flow rates of $0.3,0.5,0.8$ and $1.0 \mathrm{ml} / \mathrm{min}$ as a control. The chromatograms are shown in Fig. 2 as the thick line. The integrated area (mass recovery) and total activity (measured off-line) were summarized in Fig $3 \mathrm{~A}$ as a function of eluent rate. It clearly indicates in Fig. $3 \mathrm{~A}$ that the mass recovery increased with the increasing 

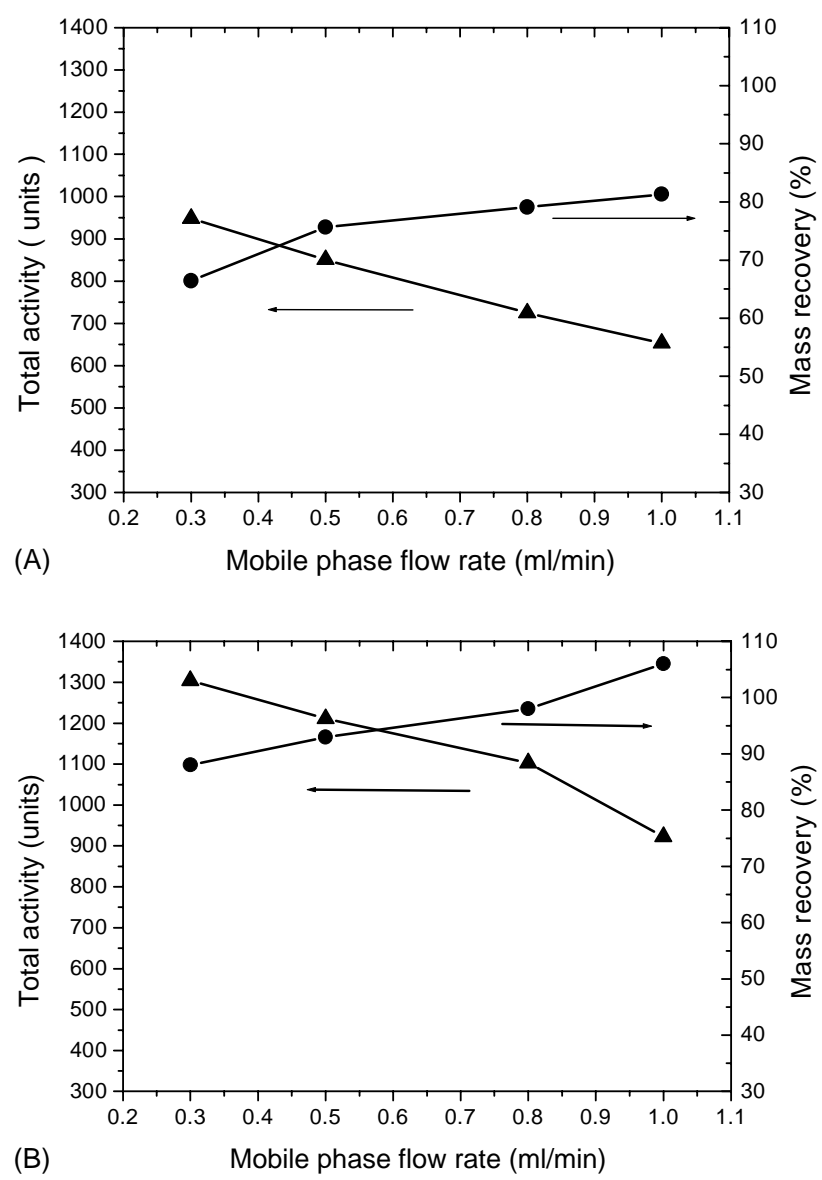

Fig. 3. Effect of flow rate in mobile phase on the protein mass recovery $(\mathbf{O})$ and total activity $(\mathbf{\Delta})$ for $(\mathrm{A})$ conventional operations and (B) chaperon solvent plug strategy. (Lysozyme loading: $20 \mu \mathrm{l}$ of $5 \mathrm{~g} / \mathrm{l}$.)

mobile phase flow rate, while the total activity decreased rapidly. The decrease of total activity with the increasing flow rate may attribute to insufficient refolding time (residence time). Therefore, it needs more time (slower flow rate of mobile phase) for denatured protein to stay inside of the SEC column for high activity recovery. However, the mass recovery decreased when mobile phase flow rate decreased on the other hand. Because partially refolded lysozyme with exposed hydrophobic patches would be very susceptible to aggregation prior to the column inlet, where no protection by packing materials of SEC was available. It was suspected that the loss of mass at slow eluent rate might be due to the protein aggregation. And the aggregation perhaps occurred immediately after the contact between the denatured lysozyme (injected sample) and refolding buffer (mobile phase). Therefore, high flow rates could possibly make the denatured lysozyme flow quickly from injector into column, and also increase the dispersion to avoid the collision of those partially refolded proteins. Consequently, mass recovery increased with increasing mobile phase flow rate. Besides, it is worth noting that the mass recovery reached somewhat a saturation value around $81 \%$. This seems to imply that mobile phase flow rate higher than $0.8 \mathrm{ml} / \mathrm{min}$ was not beneficial in terms of mass recovery in this particular case.

\subsection{Aggregation formation between injector and column inlet}

As mentioned in Fig 3A, it was noted that high mobile phase flow rate could alleviate aggregation between the injector and the column inlet to some extent. To further prove this hypothesis and evaluate the effect of chaperon solvent plug on aggregate inhibition, stream before column inlet was directly connected to the detector monitoring for aggregates. That is, the SEC column was temperately removed and the detector was set at $450 \mathrm{~nm}$ for aggregates measurement. The results were depicted in Fig. 4A for conventional operation at flow rates of 0.5 and $1 \mathrm{ml} / \mathrm{min}$. It revealed that amount of aggregate decreased as mobile phase flow rate increased. This confirms the hypothesis that high flow rate could reduce the formation of aggregates which occurred before column inlet. On the other hand, the amount of aggregates with the chaperon solvent plug were relatively insignificant as indicated in Fig. 4B. The reduction of aggregates by the chaperon solvent plug was obvious especially for low mobile phase flow rate $(0.5 \mathrm{ml} / \mathrm{min})$. It is apparent that such a chaperon solvent plug strategy offers an effective way to reduce the aggregation between injector and column inlet. Therefore, higher mass recovery is possible with slow flow rate by chaperon plug strategy. And hopefully, low mobile phase rate would provide enough residence time in the column, resulting in good refolding activity.

\subsection{Refolding of lysozyme with chaperon solvent plug strategy in SEC}

Based upon the chromatogram shown in Fig. 4A, the aggregation formation between injector and column could be circumvented to some extent with high mobile phase flow rate. Unfortunately, the result of Fig. 3A revealed that total activity recovery decreased with high flow rate. Hence, to improve the mass recovery by minimizing aggregation and enhance the total activity simultaneously would be important in the SEC refolding process. Moreover, as shown in Fig. 4B, very little aggregation was observed between the injector and column with a chaperon solvent plug. Therefore, chaperon solvent plug strategy was investigated for various flow rates to see if good mass and activity recoveries can be obtained simultaneously. The chromatograms are shown in Fig. 2 as thin lines for clear comparison with conventional operation. It was quite obvious that peak area (mass recovery) of refolded lysozyme increased significantly with the chaperon solvent plug strategy. The results were also summarized in Fig. 3B as mass recovery and total activity versus mobile phase flow rate. It is noted in Fig. 3B that high mass recovery ranging from 90 to $110 \%$ could be obtained with the chaperon solvent plug strategy. Even with low mobile phase rates as $0.3 \mathrm{ml} / \mathrm{min}, 90 \%$ mass recovery was obtained 


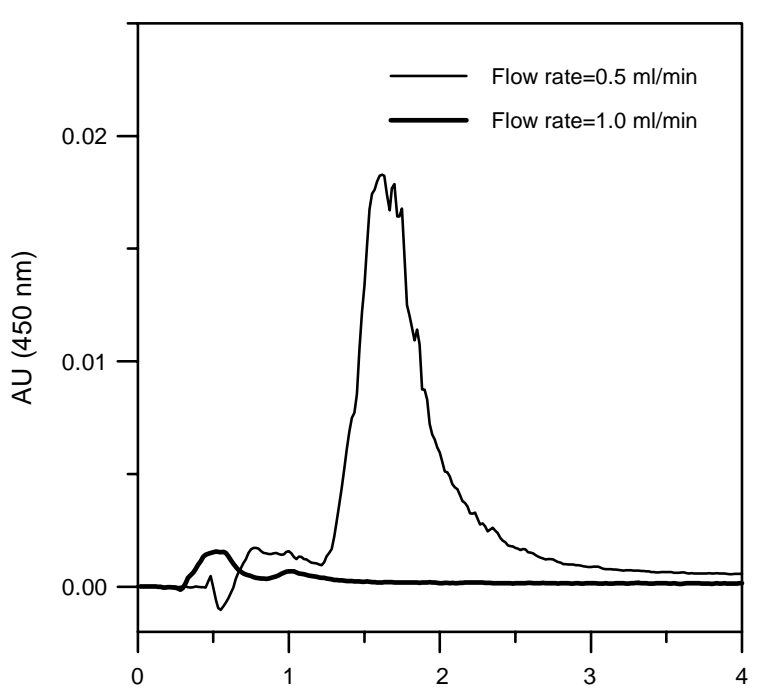

(A)

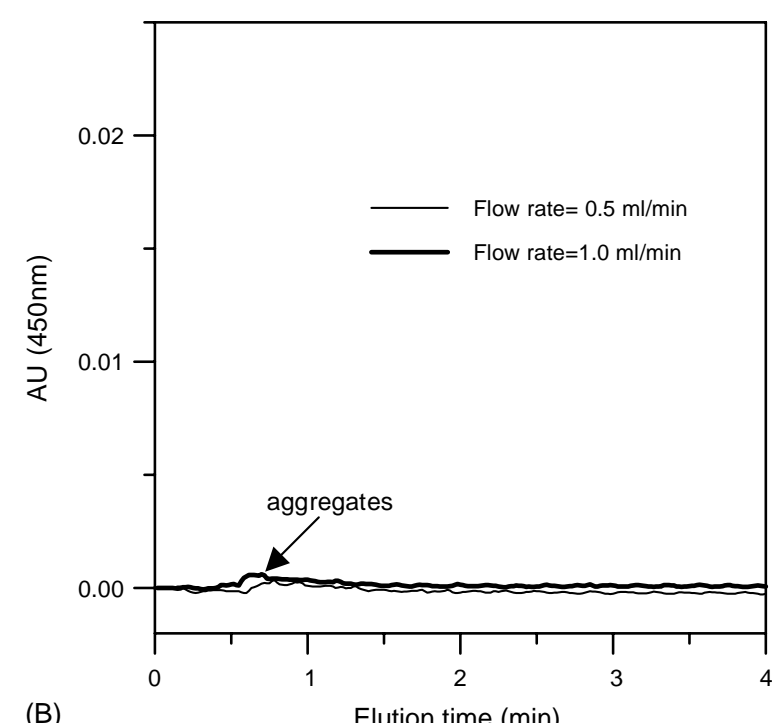

Fig. 4. Effect of flow rate in mobile phase on aggregates $(450 \mathrm{~nm})$ before the inlet of SEC column. (A) Conventional operations. (B) The chaperon solvent strategy. (Lysozyme loading: $20 \mu \mathrm{l}$ of $5 \mathrm{~g} / \mathrm{l}$.)

compared with $65 \%$ by conventional SEC. Overall, there was a significant improvement compared with $65-80 \%$ mass recovery in conventional operations. Meanwhile, the total activity recovery maintained a similar tendency, i.e. decreasing with the increasing the mobile phase flow rate, but enhanced roughly about $30-60 \%$ compared with conventional SEC. These results elucidated that the chaperon solvent plug strategy could enhance protein refolding performance, both mass recovery and total activity recovery.

\section{Discussion and conclusion}

The major challenge in optimizing renaturation processes is to prevent the formation of inactive and aggregated species in off-pathway. The aggregation reaction rate usually exhibits higher order than that of the correct folding rate [5]. This suggests that the refolding at high protein concentration would inevitably favor aggregates. The recent established SEC refolding operation can perform the buffer exchange to facilitate protein refolding/renaturation. However, aggregation formation between the injector and column inlet was shown be a major obstacle to obtain high refolding performance in the SEC process. This aggregation also experimentally proved to be originated primarily from the mixing of denatured lysozyme and refolding buffer prior to the column inlet, resulting in loss of mass recovery. Although fast flow rate in mobile phase can reduce the aggregation formation prior to the column inlet, total activity significantly decreased. The decreases of total activity may attribute to insufficient residence time of protein sample in SEC column.

How to improve the mass recovery by minimizing aggregation and to enhance activity yield simultaneously was the goal of this study. Since it was noted that the aggregation formation mainly occurred between the injector and column inlets, SEC incorporating with the chaperon solvent plug strategy was developed. The operation of SEC refolding with chaperon solvent plug strategy was designed to escort the denatured protein in the aggregation inhibitor solvent ( $8 \mathrm{M}$ urea). It was then shown that very high mass recovery could be obtained ranging from 90 to $110 \%$ with the chaperon solvent plug strategy.

However, this technique can be further investigated to improve its renaturation yield. For example, the composition of chaperon solvent, and higher protein concentration can be sought. The volume of chaperon solvent plug, $333 \mu$ l, could be also further optimized. Nevertheless, the chaperon solvent plug method was demonstrated successfully to obtain high mass and activity recoveries simultaneously.

\section{Acknowledgments}

Financial support from NSC (National Science Council) is greatly acknowledged.

\section{References}

[1] De Bernardez Clark E. Protein refolding for industrial processes. Curr Opin Biotechnol 2001;12:202-7.

[2] Thatcher DR, Hitchock A. Protein folding in biotechnology. In: Pain RH, editor. Mechanisms of protein folding. New York: Oxford University Press; 1994. p. 229-61.

[3] Mitraki A, King J. Protein folding intermediates and inclusion body formation. Biotechnology 1989;7:690-7.

[4] Goldberg ME, Rudolph R, Jaenicke R. A kinetic study of the competition between renaturation, and aggregation during the refolding of denatured-reduced egg white lysozyme. Biochemistry 1991;30:2790-7.

[5] Maeda Y, Koga H, Yamada H, Ueda T, Imoto T. Effective renaturation of reduced lysozyme by gentle removal of urea. Protein Eng 1995;8:201-5. 
[6] Gallant SR, Kundu A, Cramer SM. Modeling non-linear elution of proteins in ion-exchange chromatography. J Chromatogr A 1995;702: $125-42$.

[7] Dong XY, Yang H, Sun Y. Lysozyme refolding with immobilized GroEL column chromatography. J Chromatogr A 2000;878:197-204.

[8] Batas B, Chaudhuri JB. Protein refolding at high concentration using size-exclusion chromatography. Biotechnol Bioeng 1996;50:16-23.

[9] Hamaker KH, Liu J, Hamaker KH, Seely RJ, Ladisch CM, Ladish MR. Chromatography for rapid buffer exchange and refolding of secretory leukocyte protease inhibitor. Biotechnol Prog 1996;12: 184-9.

[10] Fahey EM, Chaudhuri JB, Binding P. Refolding and purification of urokinase plasminogen activator fragment by chromatography. J Chromatogr B 2000;737:225-35.

[11] Fahey E, Chaudhuri JB. Molecular characterisation of size exclusion chromatography refolded urokinase-plasminogen activator. Chem Eng Sci 2001;56:4971-8.

[12] Müller C, Rinas U. Renaturation of heterodimeric platelet-derived growth factor from inclusion bodies of recombinant Escherichia coli using size exclusion chromatography. J Chromatogr A 1999;855:20313.

[13] Fahey EM, Chaudhuri JB, Binding P. Refolding of low molecular weight urokinase plasminogen activator by dilution and size exclusion chromatography: a comparative study. Sep Sci Technol 2000;35:1743-60.

[14] Gu Z, Su Z, Janson J-Ch. Urea gradient size-exclusion chromatography enhanced the yield of lysozyme refolding. J Chromatogr A 2001;918:311-8.

[15] Hevenhan DL, De Bernardez Clark E. Oxidative renaturation of lysozyme at high concentration. Biotechnol Bioeng 1997;54:221-30.

[16] Wetlaufer DB, Xie Y. Control of aggregation in protein refolding: a variety of surfactants promote renaturation of carbonic anhydrase II. Protein Sci 1995;4:1535-43.

[17] Campbell MK. The three-dimensional structure of protein. Biochemistry. Saunders College Publishing; 1995. p. 86-118.

[18] Nölting B. Experimental problems of the kinetic and structural resolution of reactions that involve proteins. Protein folding kinetic. Berlin: Springer-Verlag; 1999. p. 125-35. 